\title{
LA PROTECCION DE LA NATURALEZA EN LA PLANIFICACION TERRITORIAL
}

\author{
719:502.7 (46. Valencia)
}

por

\section{Ignacio Docavo Alberti}

Vicepresidente de la Diputación Provincial de Valencia Catedrático Director del Departamento de Zoología y Biología de la Facultad de Ciencias

SUMARIO: I. METODOLOGIA GENERAL Y SU APLICACION A UN CASO CONCRETO: 1. LAS UNIDADES AMBIENTALES, SU DELIMITACIÓN Y VALORACIÓN. 2. IMPACTOS DE USO, AREAS DE DIAGNOSTICO, VALORES CRITIcos. 3. PROTECCION DE COTAS ALTAS, ESPARCIMIENTOS DE MONTAÑA.-II. CONDICIONES DEL MEDIO FISICO EN LA PROVINCIA DE VALENCIA: 1. ZONIFICACION EN AREAS HOMOGÉNEAS Y SU VALORACION. 2.

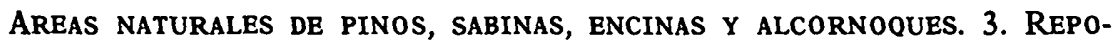
blados, matorRales, areas agrfColas y embalses. 4. ZoNaS paNtaNoSAS, DUNAS Y OTRAS.-III. CONCLUSIONES.

\section{METODOLOGIA GENERAL Y SU APLICACION A UN CASO CONCRETO}

1. LAS UNIDADES AMBIENTALES, SU DELIMITACIÓN Y VALORACIÓN

El fin que se pretende es establecer las restricciones de uso que, en mayor o menor grado, imponen al medio natural el asentamiento de nuevas urbanizaciones o instalaciones turísticas e industriales.

Lo primero a realizar es dividir el espacio que se quiere proteger, por ejemplo una Provincia, en una serie de zonas homogéneas, desde el punto de vista de la calidad ambiental, en donde se 
dan unas caracter'sticas respectivas de topografía, suelo y vegetación. Estas áreas son las que se denominan unidades ambientales.

La determinación de estas unidades y de otras particularidades que actúan como condicionantes del medio físico o natural en la planificación requiere la intervención de un complejo equipo de expertos en los siguientes campos: ecología, geología, geomorfología, hidrología, edafología, paleontología, climatología, así como en esparcimiento y paisajismo.

Este equipo ha de trabajar de forma coordinada e integradora para desarrollar una serie de estudios básicos, que se realizan cada uno en las siguientes etapas: a) Prospección del territorio; $b$ ) Delimitación de las unidades homogéneas o ambientales; $c$ ) Valoración de cad unidad, y d) Diagnóstico de la respuesta de cada una de estas unidades o áreas homogéneas a los diferentes usos: asentamientos industriales, turísticos y urbanos de distinto tipo o bien su destino para Parque Natural, reserva ecológica, etc.

En la valoración de cada unidad ambiental se distinguen distintos tipos de valores, que son los que se relacionan con el esparcimiento, conservación, productividad, culturales y estéticos, así como aquellos ligados con la contaminación y erósión. El establecimiento de estas unidades ambientales y sus valoraciones suporre un complejo y especializado trabajo.

Vamos a dar una idea de cómo se opera con ellas y de los importantísimos resultados que para la planificación territorial se alcanzan, haciendo una síntesis de la metodología seguida en el Plan de Protección del Medio Físico de la Provincia de Madrid, realizado a iniciativa del Area Metropolitana de Madrid por COPLACO.

Se han establecido en este estudio 382 unidades ambientales, describiéndose en cada una de estas unidades 42 aspectos diferentes que contribuyen al conocimiento de su calidad ambiental. En estos aspectos se incluyen los geológicos, climáticos, de contaminación, suelo, productividad, biocenosis animales y vegetales, sucesión vegetal, diversidad, fragilidad de la unidad referida a sus ecosistemas, potencial agrícola, caza y pesca, esparcimiento, interés educativo, estética del medio físico y biológico, paisaje, etc.

Cada unidad resulta con un valor actual $V_{a}$ que se puede representar por un vector de cada uno de los conceptos de valores antes indicados. La valoración de estos vectores $V_{i}$ se puede establecer por distintos procedimientos, que no es objeto de este trabajo de 
divulgación detallar aquí. Sólo diremos que cada componente $\mathrm{V}_{\mathrm{i}}$ puede, por ejemplo, compararse con una escala de referencia formada por una muestra de unidades ambientales.

Esta escala, establecida por los expertos, está dividida en rangos de 1 a 9 y permite su aplicación sistemática y consistente a todo el ámbito del proyecto evaluando los componentes del vector $V_{a}$ mediante el equipo interdisciplinario.

Pero la calidad actual $V_{a}$ de una unidad ambiental determinada puede variar bajo la influencia de las actividades humanas, bien deteriorándose, bien mejorando o aumentando de valor. Esta fluctuación del vector $\mathrm{V}_{\mathrm{a}}$, que va adquiriendo, según diferentes usos hipotéticos a que sometemos sucesivamente a la unidad ambiental, unos nuevos valores llamados $V_{f 1}, V_{f 2}, V_{f 3}, V_{f n}$ que llevan al concepto del vector de impacto $V$, que puede definirse por la diferencia $\mathrm{V}=\mathrm{V}_{\mathrm{f}}-\mathrm{V}_{\mathrm{a}}$. Los valores $\mathrm{V}_{\mathrm{f}}$ se hallan mediante las llamadas matrices de impacto, cuyos elementos describen mediante números negativos las influencias adversas que sufren los valores medioambientales $V_{i}$ por el uso del suelo. A cada uno de estos usos corresponde una matriz de impacto $P$, cuyo desarrollo nos dará el vector $V_{f}$

En el estudio desarrollado por COPLACO se han considerado para cada unidad ambiental seis destinos o hipótesis que nos darán seis valores de vector $V_{f}$ que por diferencia con el valor o impacto actual $\mathrm{V}_{\mathrm{a}}$ nos dará el conocimiento de lo que sucede con el área sometiéndola a estos seis distintos usos $\mathrm{y}$, por tanto, si disminuye o aumenta su valor con estos destinos. Las seis hipótesis de destino que se consideran para cada unidad ambiental son las siguientes: 1. ${ }^{\mathrm{a}}$ Urbanización de alta densidad. 2." Urbanización de baja densidad. 3." Parque industrial. 4." Parques suburbanos 0 espacios libres ajardinados. 5. Reserva Parcial, Parque Natural o Regional. 6." Reserva ecológica.

Si aplicamos, por ejemplo, las matrices de impacto a la unidad ambiental núm. 3 de la Provincia de Madrid, definida y valorada por COPLACO, nos encontramos lo siguiente: Valor actual $=\mathrm{V}_{\mathrm{a}}=$ $=45$; para la hipótesis $10^{\mathrm{s}} \mathrm{V}_{\mathrm{f} 1}=-35$; para $\mathrm{V}_{\mathrm{f} 2}=-18$; para $\mathrm{V}_{\mathrm{f} 3}=-33$; para $\mathrm{V}_{\mathrm{f} 4}=-6$; para $\mathrm{V}_{\mathrm{fs}}=1$, y para $\mathrm{V}_{\mathrm{f} 6}=2$.

El planificador, a la vista de la consecuencia de estos impactos, puede deducir que la unidad ambiental núm. 2 o bien se deja como en la actualidad (momento en que se realizó el estudio), o bien pue- 
de destinarse a Parque Natural o a la Reserva ecológica, pero de ningún modo a urbanizaciones o a la industria, ya que su valor se reduciría a la cuarta parte, aproximadamente en dos casos, y notablemente cuando se dedicara a urbanizaciones de baja densidad.

\section{IMPACTOS DE USO, AREAS DE DIAGNÓSTICO, VALORES CRITICOS}

Teniendo, pues, valoradas las 382 unidades ambientales y sometidas cada una de ellas a las seis hipótesis antes citadas tendremos valorados también, como en el caso anterior, los correspondientes impactos. La Administración puede así decidir conscientemente el sacrificio que supone para el medio natural un asentamiento determinado que disminuya su valor. Sólo en aras de un alto interés nacional o provincial podrá sacrificarse para la urbanización o a la industria una unidad ambiental de interés ecológico o de productividad agrícola elevada.

Es vergonzoso el asentamiento de industrias o núcleos urbanos en áreas agrícolas de gran valor productivo, mientras, por otra parte, se han desecado extensas zonas húmedas interiores o litorales de gran importancia biológica para dedicarlas al cultivo. Esto es un contrasentido que a la larga tiene que pagar nuestro país a alto precio. En la práctica el estudio puede simplificarse, ya que existen numerosas unidades ambientales que se comportan de una manera semejante ante los impactos de las actividades humanas que se consideran. Por ello, estas unidades ambientales, que responden de modo semejante a los impactos, se agrupan en las llamadas áreas de diagnóstico. En la Provincia de Madrid las 382 unidades ambientales se han agrupado en 28 tipos de áreas de diagnóstico, que a su vez se han clasificado en ocho grupos diferentes atendiendo a criterios ecológicos, estético-culturales y de productividad. Estos grupos se expresan por números romanos, del I al VIII, y los tipos reunidos en cada uno, por una letra mayúscula que sigue al número del grupo. Así, dentro del grupo I se distinguen las áreas de diagnóstico I.A., I.B., I.D., etc., hasta cinco.

Pero la valoración que se ha obtenido de una unidad ambiental o de un área de diagnóstico mediante las matrices de impacto, se hace en un principio independientemente de otras unidades contiguas que podrían verse afectadas en su valor por el uso que les 
‘demos, aunque sea uno adecuado a ellas. Así, por ejemplo, una unidad ambiental de secano de nulo interés ecológico y escasa productividad agrícola podría, según su valoración en impactos, ser destinada a urbanizaciones de baja densidad; pero si esta unidad resulta ser una cuenca de vertido hacia un lago o embalse para agua potable, exige una restricción mayor de uso, y esto es preciso aplicarlo mediante el concepto de los llamados aspectos críticos, siendo uno de ellos el vertido que se acaba de explicar.

Entre los aspectos críticos, que se indican por letras minúsculas que se colocan como subíndices de las letras mayúsculas con que se definen las agrupaciones de áreas de diagnóstico, por ejemplo $\mathrm{IA}_{\mathrm{h}}$, resulta restringido el uso del área de diagnóstico IA, por indicar ${ }_{h}$ lugares de interés naturalístico especial (ecosistemas relictos de enorme interés en la conservación o sitios de interés paleontológico o faunístico especial).

rr) indica zonas de vertido inmediato sobre masas de agua de interés;

c) áreas que contaminan aguas subterráneas;

d) poca capacidad de dispersión de contaminantes aéreos y, por tanto, restricción del uso para industrias contaminantes de la atmósfera, aunque admitirá en principio tal asentamiento un área de diagnóstico del mismo valor, pero que no tuviese este aspecto. crítico de poca dispersión de contaminantes.

Existen otros varios aspectos que no vamos a detallar, pues lo explicado basta para tener el concepto, que es lo que aquí interesa.

En el estudio de COPLACO se representan en un mapa a escala 1 : 100.000 las unidades de diagnóstico y los niveles máximos de acogida de actividades, fijándose para cada tipo de zona con capacidad similar la correspondiente normativa para su obligada consideración en el planteamiento urbanístico subsiguiente.

El grado de actuación o nivel de uso recomendable para cada área de diagnóstico se especifica mediante una jerarquía de nueve niveles sucesivo de uso, que va desde el equivalente a un uso exclusivamente científico, sin permitir ningún tipo de preservación. La asignación a cada área de diagnóstico de los niveles de uso para ella recomendables se realiza a través de la matriz de diagnóstico, en la que se clasifica cada área según el criterio de mayor relevancia desde el punto de vista de la conservación de sus valores más importantes, ya sean de tipo naturalístico, estético o de productividad, señalándose los niveles máximos de uso recomendable. 
En el mapa se señalan también las zonas de interés geológico, paleontológico y didáctico, dándose las normas a seguir para la conservación mediante una clasificación conveniente. En estas zonas se sitúan, mediante signos convencionales, las localizaciones exactas de yacimientos de fósiles mineralógicos de interés, geomorfológicos, estratigráficos, etc.

Este Plan Especial del Medio Físico del Area Metropolitana constituye un estudio muy complejo respecto a lo que supone un conjunto de directrices de planeamiento en orden a la conservación de los valores ecológicos, culturales y productivos de la Provincia de Madrid y a la defensa del medio natural frente a los agentes contaminantes. Tiene el carácter de directrices vinculantes para la redacción del planeamiento territorial, con las excepciones que se indican en las normas generales del Plan, aprobado por Orden ministerial de 27 de octubre de 1975.

En el apéndice figura también el Catálogo de Protecciones existentes, según la legislación específica de los Ministerios de Agricultura y del de Educación y Ciencia, de protecciones decretadas en relación con los embalses, así como un mapa de los Montes de Utilidad Pública del Estado y consorciados. También se incluye un Catálogo de Protecciones propuestas con arreglo al artículo 20 de la Ley del Suelo de 1956, hoy artículo 25 en la nueva, y que incluye sitios de interés naturalístico, geológico o paleontológico que deben ser objeto de una protección total, tal como el Plan especifica en los mapas de áreas de diagnóstico y capacidades de acogida.

\section{PROTECCIÓN DE COTAS ALTAS, ESPARCiMIENTOS DE MONTAÑA}

Mención especial merece que las superficies de cotas altas, con relación a las alturas medias de la Provincia, aparecen punteadas en el mapa y orladas por puntos gruesos para señalar que estas zonas son objeto de una protección muy especial con respecto a la conservación, y es que estas zonas altas tienen por lo general un interés grande desde el punto de vista ecológico (botánico, faunístico, geológico, etc.), y también paisajístico, y son asimismo paisajes escénicos emisores o receptores de vista. Por tanto, está justificadísimo que estas zonas se quieran conservar y sean objeto de una protección especial. Sería ilógica la construcción de una urbanización en una superficie anterior a miradores o puntos dominantes 
emisores de vista desde los que se divisan amplias panorámicas, pues ello sería sustraerlos al disfrute público general ante la incomodidad de atravesar una zona privada o dar molestos rodeos, o también, si a su vez estos puntos son receptores de vista, el deterioro supondría para el paisaje ver tras ellos construcciones del tipo que fuese.

En las zonas no calificadas como de protección ecológica total por su interés científico, sino de protección más laxa, caben los esparcimientos y el disfrute por un número de visitantes que será reglado en la ordenación de la zona. Los esparcimientos pueden ser con limitaciones más restringidas o de primer grado o con limitaciones menores, que son los esparcimientos con restricciones de segundo grado. Precisamente en los esparcimientos de montaña puede haber en los lugares convenientes, según las normas del Plan, determinadas construcciones, a razón de un metro cúbico por cada cinco metros cuadrados, pero la unidad mínima de planeamiento será de 500 hectáreas, concentrándose el volumen de edificación en áreas no superiores a 15 hectáreas de extensión superficial cada una y cuya suma total no excederá del 15 por 100 de la superficie total ordenada. Si la unidad fuese de la mínima superficie admitida, 500 hectáreas, sólo cabría asentar cinco unidades urbanizables de 15 hectáreas cada una. Además tendrían el carácter de ordenación de los Planes de Interés Turístico Nacional, y para iniciar el expediente el planificador tendría que tener la conformidad del Area Metropolitana. Del supuesto de las 500 hectáreas, el propietario de las mismas deberá ceder, libre de cargas y en pleno dominio, el 50 por 100 de la superficie réstante no incluida en unidades urbanizables al Ayuntamiento correspondiente (en el caso supuesto 212,5 hectáreas) para su inclusión en el Catálogo de Montes de Utilidad Pública. Toda la superficie no incluida en las unidades urbanizables (en este caso las 425 hectáreas) será calificada de espacios libres sujetos, en cuanto a su alteración, al régimen previsto en la Ley de 2 de diciembre de 1963 sobre zonas verdes y espacios libres protegidos. Mas en el Plan se asegura el cumplimiento de no edificabilidad de la superficie de cesión (las 212,5 hectáreas) ni del resto, obligando a hacer constar en el Registro de la Propiedad, con carácter previo a la concesión de las licencias a que se refiere el artículo 178 de la Ley del Suelo, la inedificabilidad de tales espacios. Vemos, pues, a través de estas normas de edificaciones de esparcimiento en montaña lo lejos que estaría alguna recientemen- 
te pretendida urbanización como centro de interés turístico nacional de cumplir estas condiciones y la plena justificación de la oposición encontrada, según las condiciones puestas a la construcción en montañas de interés ecológico, paisajístico y escénico, pero en las que puede permitirse el esparcimiento.

Por otro lado, es muy interesante señalar el anillo o cinturón verde que se ha proyectado a lo largo de todo el perímetro de la ciudad de Madrid, verdadera barrera defensiva contra la contaminación y pulmón verde periférico y próximo a la gran urbe.

Ciertamente que tan complejo estudio ha supuesto la inversión de una fuerte cantidad de dinero dado el extenso equipo interdisciplinario científico y técnico, que ha contado con la colaboración del ICONA, además de, como es natural, un equipo de juristas indispensable en toda tarea de planificación de esta indole. Un Plan especial del medio físico semejante debería iniciarse en otras Provincias españolas, especialmente en las más pobladas e industrializadas, donde la planificación de esta índole, la construcción y las urbanizaciones turísticas y de vivienda de temporada hacen incesantes estragos irreversibles en la conservación de la Naturaleza.

Naturalmente que la tarea es larga, pues no sólo es el gasto que el equipo supone, sino los elementos básicos que para tal objetivo el equipo tiene que servirse, tales como mapas de vegetación, de suelos, geológicos, paisajísticos, de ecosistemas y biocenosis, etc., lo que exige un trabajo previo, quizá largo y costoso, pues no todas las Provincias disponen de estos datos con el detalle suficiente, cuando no carecen casi en absoluto de los mismos. Si a ello unimos el tiempo de duración del estudio tendremos que concluir que, dada la velocidad con que actúan las acciones humanas destructoras, hemos de acudir a otra medida previa cautelar y urgente mientras se redacta el Plan Especial del Medio Físico de la Provincia. Tal es el Catálogo de Parques Naturales, Paisajes y Jardines que se contempla en el artículo 25 de la Ley del Suelo, Catálogo que han de aprobar las Comisiones Provinciales de Urbanismo o el Ministerio de la Vivienda y que es obligatorio para poder redactar luego los Planes especiales correspondientes a lo incluido en tal catalo. gación. 


\section{CONDICIONES DEL MEDIO FISICO EN LA PROVINCIA DE VALENCIA}

\section{ZONIFICACIÓN EN AREAS HOMOGÉNEAS Y SU VALORACION}

Precisamente para nuestra Provincia tenemos ya un estudio de los condicionantes del medio ambiente físico para la planificación industrial y urbanística. Se trata de una de las fases del trabajo «Desarrollo de la Provincia y localización del suelo industrial y equipamiento» que, promovido por la Diputación, ha sido realizado por SIE en colaboración con otras sociedades, ya que a éstas les fue adjudicado en concurso un estudio para la planificación y localización de los asentamientos industriales en la Provincia, cuya segunda fase ha sido ya entregada a la Corporación.

Ciñéndonos a lo que en el aspecto de la conservación nos interesa, que son las condiciones del medio físico, haremos una sintesis de este trabajo, que se atiene, en líneas generales, a la metodología descrita anteriormente y desarrollada por COPLACO en el estudio del medio ambiente físico de la Provincia de Madrid como condicionante de las acciones de planificación. La filosofía o principio que subyace en el estudio es la misma, naturalmente, que ya hemos desarrollado: impedir cualquier deterioro irreversible del medio natural (en este caso el valenciano) más allá de límites tolerables.

Por carecer de tiempo, medios económicos y trabajos previos de base, como mapas de vegetación, de fauna, suelo, yacimientos paleontológicos y geológicos, de biocenosis, etc., de la Provincia, el estudio es mucho menos detallado que el de COPLACO, pero representa un importante esbozo que, completado por un equipo técnico apropiado que disponga de suficiente tiempo y ayudas económicas, puede convertirse en un trabajo detallado y complejo como el realizado en Madrid sobre los condicionantes del medio físico en la planificación territorial.

Se ha comenzado por realizar una zonificación de la Provincia en áreas relativamente homogéneas, es decir, que cada una de ellas presenta en todos sus puntos indicadores biológicos e inertes iguales o considerados como equivalente a los efectos del trabajo.

Se han definido 21 de estas áreas, que resultan, a la escala utilizada, amplias y muy contrastadas y son subdivisibles en otras más 
pequeñas a menos escala. Estas áreas pueden asimilarse a ecosistemas, los que constituyen la parte fácilmente perceptible por sus manifestaciones externas más notables y características. Los principales indicadores utilizados para el establecimiento de estas áreas han sido la vegetación natural, los cultivos, la geomorfología, la calidad de los suelos y la estética del paisaje.

En realidad estas áreas equivalen a las llamadas de diagnóstico en el estudio de COPLACO, aunque no se ha llegado a ellas por la división del territorio en las unidades ambientales, su valoración y agrupamiento en las áreas del diagnóstico de todas aquellas que se portaban de modo semejante ante las acciones humanas de planificación o impactos. Ello hubiera exigido un trabajo mucho más largo y enjundioso, llegándose por el método empleado a la suposición de que estas áreas pueden comportarse a grandes rasgos como las de diagnóstico, aunque conscientes que estudio más detallado mediante unidades ambientales podrá subdividir a estas 21 zonas en otras más reducidas y homogéneas.

Una vez mapificada y descrita la zonificación, se realiza su valoración según una escala jerárquica que varía de cero a diez. La valoración se establece independientemente para cada uno de los conceptos y dimensiones que constituyen la calidad ambiental de los ecosistemas, de los que se han considerado en el estudio el valor naturalístico, de productividad y valor paisajístico, de un modo semejante a la metodología antes descrita.

Definidas, descritas y valoradas las áreas de diagnóstico $u$ homogéneas antes aludidas, se establecen los usos que toleran dentro de una serie de ellos establecida, es decir, su comportamiento ante los impactos dentro de los límites tolerables que no lleven a ningún deterioro. Estos usos establecidos son: «Restringido de primer grado», «Restringido de segundo grado", "Ocupación limitada del suelo», "Urbanización dispersa», "Urbanización de media densidad» y «Urbanización de alta densidad».

Independientemente de esta tolerancia de usos se establecen los aspectos críticos, ya que pueden modificar los usos en determinados casos haciéndolos más restringidos, considerando aquí solamente dos, que se refieren a la capacidad dispersante de la atmósfera y a la vulnerabilidad a la contaminación de los acuíferos subterráneos.

El estudio consta de cinco mapas: uno, de las 21 áreas homo. géneas definidas y su situación en la Provincia; otro, de la capaci- 
dad de usos que ofrecen dichas áreas dentro de límites tolerables. Un tercer mapa, con la capacidad dispersante de la atmófera, clasificando la Provincia en una zonificación de capacidad diferente: buena, escasa y regular. A continuación viene un cuarto mapa de zonificación por la capacidad de contaminación de los acuíferos subterráneos, calificados como de contaminación alta, media, variable y baja. Terminando el estudio con un mapa de montes catalogados de propuesta de espacios naturales protegidos.

Todos los aspectos del trabajo aquí sintetizados son estudiados con detalle a lo largo de las 40 páginas del resto del estudio y de los mapas.

Ampliamos aquí ahora algunos aspectos de los enunciados. Al hablar de las áreas se indica: «Es preferbile hablar de tipos de paisaje en vez de tipos de sistemas o ecosistemas, ya que su identificación se hace en base a unos signos externos y conspicuos del sistema de relaciones subyacentes fácilmente perceptibles".

Dentro de las zonas de carácter forestal y, en general, donde no existe ningún tipo de agricultura se ha utilizado como indicador principal la vegetación (pinares, sabinares, encinares, matorrales, etcétera). En otros casos, como las zonas húmedas, ha predominado el criterio geológico al definir las distintas unidades. En los espacios dedicados a la agricultura se ha utilizado como indicador principal la calidad de los suelos en base a su potencialidad agraria, clasificándolos en tres grupos que dan lugar a otras tantas unidades o áreas: a) aquellos suelos que soportan una agricultura excelente; $b$ ) aquellos que por una circunstancia fácil de paliar soportan por el momento una agricultura inferior a la que les corresponde, siendo en general suelos de secano, y $c$ ) suelos que por diversas circunstancias ambientales mantienen una agricultura pobre y difícil de mejorar.

Por último, y en lo que se refiere a los valores de tipo perceptual, se delimitan con arreglo a criterios estéticos y culturales y se aplican sobre zonas que no siendo sobresalientes paisajísticas de interés, como pueden ser matorrales en posiciones topográficas re: levantes y visibles desde áreas pobladas, o bien por su proximidad a la costa, etc.

De las áreas homogéneas 17 se han delimitado atendiendo a criterios naturalísticos, tomando como base la vegetación o núcleos geomorfológicos, que al fin y al cabo determinan un tipo especial 
de vegetación; otras tres áreas responden a criterios de productividad, y una sola está basada en valores perceptuales de tipo estético-cultural.

\section{AREAS NATURALES DE PINOS, SABINAS, ENCINAS Y ALCORNOQUES}

Entre las 17 áreas de valores naturalísticos tenemos las siguientes:

\section{1." Pinares naturales de "Pinus halepensis" sobre substrato calizo}

Este pino, llamado vulgarmente carrasco, representa en la Provincia el papel más importante de las masas boscosas, tanto en calidad como en cantidad.

Puede llegar a los $1.000 \mathrm{~m}$. de altitud, pero su mejor zona es una faja próxima al litoral, de cotas inferiores a los 800 metros.

Estos pinares tienen una gran importancia desde el punto de vista ornamental, socio-recreativo y protector, ya que pueden formar bosques naturales o artificiales hasta la proximidad del mar y en las sierras áridas y desforestadas por su sobriedad y rusticidad. Sus localizaciones constituyen áreas de alto valor paisajístico, tanto por el relieve y substrato como por la masa biológica. En estos bosques deben establecerse restricciones de uso de primer grado. Comprenden éstas aquellas actividades ligadas a las utilizaciones actuales de tierras para agricultura, ganadería y selvicultura, así como los desarrollos necesarios para su ejercicio. También caben aquí utilizaciones recreativas al aire libre, sin ningún tipo de construcciones $o$ acondicionamientos fijos.

2. Pinares naturales de "Pinus pinaster» (rodeno) sobre substrato calizo o silícico

En las sierras de Utiel, Martes, Tejo, Santa María Caroche y otras crece sobre substrato calizo, mientras que en la Serranía de Chelva se asienta sobre substrato silícico.

Este pino es robusto y de crecimiento rápido, de porte elegante, mucho más que el carrasco; su aprovechamiento es notable y po- 
see valor paisajístico y estético muy sobresaliente, aumentado por el contraste en colorido que da en nuestras sierras calizas. Las áreas señaladas en el estudio incluyen enclaves pequeños de pino carrasco.

En estos pinares el uso permitido es como en los del área an. terior.

\section{3." Pinares de "Pinus laricio» (pino negro) introducido}

Se encuentra en la cuenca alta del Turia, donde se ha extendido artificialmente como continuación de su vegetación espontánea en las montañas del Maestrazgo, que constituyen su habitat natural.

La especie es de crecimiento lento y delicado, aunque de gran longevidad, prefiriendo substrato calizo, y se instala entre los 800 y los $2.000 \mathrm{~m}$. de altitud en ubicaciones de ladera.

Sus asentamientos tienen un alto valor estético naturalístico. El uso recomendado para esta área es como el de las anteriores, restricción en primer grado.

\section{4." Pinares naturales de "Pinus sylvestris» (pino silvestre o albar)}

Apenas se encuentran pinares de esta especie en nuestra Provincia debido a unas exigencias ecológicas que muchas veces no se dan en nuestro suelo.

Las manchas más importantes están en la Sierra de la Puebla de San Miguel (Rincón de Ademuz), aunque aparece dominado por el pino negro en muchos puntos.

Estas áreas ofrecen un carácter estético-social y de recreo que va en aumento y constituye un paisaje montañoso de alta calidad intrínseca, siendo muy interesante su fauna, que es característica y compleja, como corresponde al carácter íntegro del ecosistema.

Las manchas señaladas incluyen algunos enclaves de roble negral (Quercus pirenaica), y en el fondo de valles, árboles caducifolios.

Las restricciones de uso son de primer grado. 
5." Pino carrasco ( P. halepensis») sobre arenales $y$ dunas costeras

La mejor representación de la especie en este enclave la tenemos en la Dehesa de El Saler. Es éste un ecosistema de excepcional valor por la estabilidad y equilibrio existente entre su comunidad biótica y el substrato físico en que se establece. Es además muy frágil, principalmente por la poca superficie que ocupa, sobre todo en situaciones urbanizadoras. No nos extenderemos más, pues el ecosistema de El Saler ha sido tratado por nosotros en multitud de artículos de prensa e informes en congresos nacionales e internacionales. Sus múltiples valores han sido destruidos en gran parte, haciendo caso omiso de las advertencias de los biólogos desde el año 1966. La desgracia podrá servir al menos de ejemplo visible y candente para actuaciones futuras, como las que se realizan y pretenden en Sierra Calderona y en otros puntos de la Provincia.

\section{6." Sabinares de "Juniperus thurifera»}

La sabina albar es una importante especie, pues se trata de un fósil viviente, de una reliquia botánica de los tiempos del terciario. Por ello se mantiene en un delicado equilibrio con el medio, roto el cual se produce su desaparición. Esta es prácticamente irreversible.

Se sitúa en altas mesetas y páramos de 800 a $1.000 \mathrm{~m}$. de altitud con clima mediterráneo árido y continental frío. Se suele situar en los calizos jurásicos y areniscas del Bunter

En Ademuz encontramos restos de bosques de sabina en Sierra Tortajada, que son estribaciones de un área central situada en la Sierra de Albarracín. Dado su carácter relicto, se propugna su conservación a toda costa, igual que los testimonios artísticos humanos de otras épocas, pues además de un valor científico y ecológico, tras su desaparición sólo quedará un suelo desierto debido a la erosión en la mayoría de los casos.

Sus áreas deben constituir reservas integrales con máxima protección. 


\section{7." Encinares de "Quercus ilex" (encina común)}

De esta especie no quedan sino bosquetes aislados y en ocasiones adehesados, sin muchas características del bosque primitivo debido a la fuerte intervención humana, que muchas veces ha reducido los encinares a monte bajo.

Forman el más característico paisaje español y su supresión debe evitarse además por otras muchas razones en cuyo detalle no vamos a entrar aquí.

Muy frágil ante los procesos de urbanización, puede tolerar actividades recreativas. Paisaje de gran calidad y característico del país.

Su área incluye enclaves del quejigo (Quercus lusitanica), uso limitado a restricciones de primer o segundo grado. Estos comprenden las mismas utilizaciones que las de primer grado más instalaciones de acondicionamiento permanente, caminos o sendas, plataformas para picnic, con mesas, bancos, puentes, etc., y alguna edificación poco importante.

\section{Alcornocales}

Son formaciones del Quercus suber sobre substrato silícico, ubicados fundamentalmente en Portacoeli, en contacto con Pinus halepensis. Se presenta en ejemplares aislados o en pequeñas manchas, pero en mucha menos cantidad que la encina.

Son comunidades climáticas de alto valor ecológico y paisajístico.

Deben constituir sus enclaves reservas integrales de máxima protección y restricción de usos.

3. Repoblados, matorRales, Areas agrfcolas y embalses

9.-12. Areas de repoblaciones de pinos

Constituyen los montes repoblados, respectivamente, con las especies de pinos mencionados en las cuatro primeras áreas homogéneas antes descritas. 
El uso que permiten son el de restricciones de primero y segundo grado.

\section{Ecosistemas degradados o de sustitución}

Se trata de matorrales calcícolos o silicícolos, con especies características para cada uno y otras muchas comunes. En muchos casos tienen cierto valor ecológico, pero son de escasa producción, y sólo cuando están situado en enclaves topográficos relevantes pueden constituir un elemento paisajístico de gran consideración. Resumiremos las características de esta área homogénea diciendo que, en conjunto, tiene escaso valor ecológico, productivo y paisajístico y que en ella son posibles todos los usos, desde el restringido en el primer grado hasta la urbanización de alta densidad. El destino que se le da a los espacios ocupados por este tipo de unidad dependerá de las características que presenten en la zona a planificar. Si, por ejemplo, la situación topográfica del espacio ocupado por este tipo de paisaje es relevante, la restricción de uso sería mucho mayor que en otras situaciones menos favorables.

\section{Areas de mediano o bajo potencial agricola}

Está formada esta unidad paisajística o área homogénea por cultivos de secano, cereal, viñas, algarrobos, etc., en zonas de carácter árido con suelos de escasa productividad agrícola. En ocasiones existen circunstancias que dan a esta unidad un cierto valor cultural y paisajístico o puede tener interés para la conservación de cierta avifauna.

En estos casos tales áreas se delimitan por el criterio más destacado, que son el ecológico o el paisajístico, y su uso será más restringido. De no mediar estas circunstancias especiales, estas áreas de suelos pobres son susceptibles, como en el caso anterior, de todos los usos, es decir, que por parte de los condicionantes del medio físico, que es desde el punto de vista que aquí analizamos los destinos de uso, no hay inconveniente para que éstos sean cua. lesquiera. 


\section{Areas agricolas excelentes}

Son las que mantienen una agricultura altamente productiva. Están situadas en fondos de valle, con agua abundante. Son zonas muy conflictivas en razón de las presiones que en ellas se producen para los asentamientos urbanos e industriales.

La agricultura de estas tierras tiene su origen en tendencias heredadas de un pasado remoto, cuando el hombre no poseía medios de ocupar los páramos u otras áreas geográficas para él hostiles. Hoy día esta tendencia, con los medios mecánicos existentes, tiene difícil justificación.

La zona litoral y los suelos interiores con agua de riego constituyen un patrimonio de excepcional valor agrícola (hasta cuatro cosechas al año) que por su forma de cultivo y los procedimientos antiquísimos y originales de administración del agua constituyen además un rico patrimonio cultural que no debemos despreciar. Debemos defender a toda costa el ejemplar aprovechamiento de estos suelos por el labrador valenciano debido a su excepcional preparación y amor a la tierra. La normativa para protegerlo de tanta presión industrial y urbana debe ser dura, y sobre todo para preservar esas aguas profundas que utilizan parte de los regadíos, pues debido a la permeabilidad de los terrenos de gran parte de estas zonas su contaminación es muy fácil y se debe exigir la no instalación de cualquier tipo de industria contaminante o, al menos cuando ello no sea posible, la aplicación de tecnologías que palien la posibilidad de dicho efecto.

La competencia de estos regadíos frente al valor del suelo urbano no puede medirse con una visión a corto plazo y en términos puramente económicos. El alto precio del suelo urbano tiene sólo un carácter artificial, es decir, puramente coyuntural y no real y permanente, siendo un producto de las altas concentraciones humanas, tan discutidas hoy. La ocupación del suelo agrícola por asentamientos urbanos e industriales supone su destrucción irreversible.

Es una pena cómo vemos que esto va ocurriendo de día en día, mientras que, por otra parte, se desecan tierras de gran valor ecológico y de problemático rendimiento agrícola (como ha ocurrido en muchas zonas húmedas) para cultivos. Sería deseable que el im- 
pulso urbanizador se desarrollara sobre otras áreas homogéneas de menos valor que la aquí descrita.

Los ríos y cauces de agua de estas zonas bajas de la Provincia han perdido gran parte de su enorme valor ecológico por ser aprovechadas sus aguas para el riego, razón por la cual se han incluido en el estudio SIE en esta unidad y no en otra separada.

El uso recomendado para esta unidad es el asentamiento, con restricciones de primero y segundo grado y las definidas como ocupación limitada del suelo, que incluye los dos casos anteriores más cualquier otro que cumpla algunas condiciones que se definen en el trabajo y de los cuales las dos fundamentales son: a) Actuación sobre superficies generales. $b$ ) Que de estas superficies ocupe una pequeña proporción en núcleo compacto, garantizándose la depuración de todos los vertidos al suelo, ya que las zonas sobre las que se aplica suelen ser terrenos de aluvión muy contaminables. En el resto de la unidad de planificación, que como dijimos será extensa, quedará garantizada la continuidad del uso agrícola actual.

\section{Suelos agricolas potencialmente muy buenos}

Corresponde a aquellas áreas agrícolas cuyos suelos presentan algunos problemas subsanables con relativa facilidad y que se refieren a costras calizas, salinidad o a dificultades con el nivel freático.

Su recuperación para la agricultura productiva no es difícil. Por tanto, sus usos deberán ser análogos a los prescritos para el área anterior.

\section{Masas de agua artificiales y su entorno de vertido directo}

Se incluyen aquí los embalses más importantes de la Provincia: Generalísimo, Cofrentes, Loriguilla, Forata, Buseo y Contreras.

Todos tienen en mayor o menor grado un notable interés ecológico y paisajístico y constituyen zonas atractivas, por su calidad y accesibilidad, para el esparcimiento y recreo al aire libre de la población valenciana Existe una fauna piscícola más o menos interesante, según los casos; mamíferos, como la nutria, en ciertos 
embalses, de enorme interés por lo amenazada que está esta especie. Es de destacar también en algunos la avifauna, con especies como el ánade real (Coll vert) y el pato colorado (Sivert). Existen sobre los entornos de estos embalses presión recreativa y peligro de desarrollo urbano para segunda residencia (urbanizaciones) que deben prohibirse, al menos en las proximidades de la masa de agua. Muchas veces las aguas de estos embalses se utilizan además para el abastecimiento de poblaciones, y su peligro de contaminación es muy grave, pues los terrenos que las circundan son frecuentemente muy contaminables. Deben, pues, cuidarse las redes de desagüe y prohibirse los pozos sépticos y pozos negros. Existen también algunos problemas de aterramiento, y en el de Forata, la fuerte contaminación del río Magro debería evitarse. Es recomendable admitir aquí sólo los usos definidos dentro de los correspondientes a restricciones de primero y segundo grado.

\section{ZoNas PANTANOSAS, DUNAS Y OTRAS}

\section{Marjales, marismas y áreas pantanosas}

Nuestra franja costera es abundante en albuferas y terrenos pantanosos, cuya fertilidad en algunos puntos ha movido su transformación en arrozales y huertos.

Todos estos marjales constituyen ecosistemas de gran valor ecológico, especialmente por la multitud de aves acuáticas que dependen de ellos. La mayoría de sus especies son migratorias, y de ahí el interés internacional de estas zonas.

A la antigua y tradicional desecación de tales áreas para cultivos agrícolas, fomentada por la nefasta Ley Cambó, hoy ya fuera de lugar, se han añadido en los últimos años las destrucciones debidas a los desarrollos turísticos, industriales y de vías de comunicación, más los terribles efectos de una contaminación creciente.

Nos hemos referido en multitud de ocasiones al interés internacional de la conservación de estas zonas húmedas, objeto de convenios y proyectos internacionales, en innumerables artículos y trabajos con motivo de las nefastas acciones sobre la Dehesa de El Saler y Albufera, y volvemos ahora a insistir aquí en la gran cantidad de valores naturalísticos de todo tipo que encierran y la necesidad a toda costa de su conservación. 
Otras zonas húmedas, aparte del entorno de la Albufera, son los marjales de los Valles, Sagunto, Alboraya, los de la orilla derecha del Júcar, hasta Gandía, y el marjal de Oliva, de menor extensión que el de Gandía y, por tanto, más frágil.

Estos lugares no deben desecarse, industrializarse ni crear en ellos urbanizaciones bajo ningún concepto. También son un peligro para su existencia las grandes vías de comunicación recientemente construidas y las autopistas en construcción. El único uso admitido es el de restricciones en primer grado.

\section{Arenales y dunas litorales más o menos estabilizadas}

Son ecosistemas típicos de las costas bajas y arenosas con comunidades bióticas muy distintas a los de las costas abruptas. Por lo que respecta a la vegetación, dominan en sus niveles herbáceos las especies psammofilas y halófilas. La evolución natural de estas hierbas se dirige hacia el sabinar-pinar, pero esta evolución ha sido interrumpida por incendios, urbanizaciones y otros usos indebidos.

Numerosas comunidades de insectos de gran interés viven en estas áreas.

La valoración de este paisaje aumenta extraordinariamente cuando entran las comunidades arbóreas, pero resulta siempre alta por la presencia continua del mar.

En este área se incluyen las dunas y arenales desprovistos de grandes árboles (pino carrasco principalmente) de nuestro litoral, hoy casi destruido por los asentamientos humanos.

\section{Paisajes escénicos emisores o receptores de vista}

Comprende esta unidad, definida bajo un criterio perceptual, aquellas situaciones no incluidas en áreas de alta calificación ecológica o naturalística. Se corresponde principalmente con situaciones topográficas relevantes y determinadas áreas próximas al mar. En el primer caso, la mayor parte de estas zonas se sitúan en la rampa que procede de las montañas interiores y desciende en graderío a los llanos litorales. Esta rampa dominante posee un alto potencial de visualización sobre la llanura costera y el mar. A su vez es elemento muy visible para la densa población costera. Cuan- 
do las zonas de la antedicha rampa soportan vegetación arbórea se han incluido en las áreas homogéneas descritas bajo un concepto naturalístico, y se han incluido bajo un concepto paisajístico las que soportan matorrales o cultivos de media o baja productividad.

\section{Areas de interés naturalístico especial por aspectos parciales o totales}

Son zonas no incluidas en las áreas homogéneas antes descritas por merecer ser declaradas espacios naturales de protección especial. En muchos de estos biotopos se encuentran importantes especies de la flora y la fauna típicas de la Región. De no ser consideradas de forma especial estas áreas y sufrir modificaciones e incluso serias transformaciones, se puede destruir irreversiblemente su carácter originario.

El trabajo describe a continuación una serie de zonas que reúnen las características arriba reseñadas. Todas ellas y otras más han sido consideradas por nosotros como de inclusión en el Catálogo de Espacios Naturales Protegibles como primera medida cautelar urgente, para ser calificadas luego como Parques Naturales, Reservas, etc.

Tenemos, pues, en el tomo dedicado a los condicionantes del medio físico del estudio de SIE y colaboradores, un trabajo paralelo al desarrollado por el Area Metropolitana de Madrid para poder contar con una base destinada a establecer las restricciones de uso que en mayor o menor grado impone el medio natural al asentamiento de nuevas urbanizaciones $e$ instalaciones turísticas $e$ industriales en la Provincia de Valencia.

\section{CONCLUSIONES}

Ya hemos dicho las razones por las cuales este estudio no ha podido ser hecho con el detalle y minuciosidad con que COPLACO lo ha realizado en la Provincia de Madrid.

Naturalmente que sus conclusiones no son vinculantes jurídicamente, pero sí que representan una estructura básica de más detalle $\mathrm{y}$, por tanto, de perfeccionamiento posterior. La Diputación, junto con otros organismos de la Administración competentes en el 
tema, puede dar los pasos necesarios para que se incluyan como Plan Especial de Protección del Medio Físico de la Provincia de Valencia dentro de un Plan Director Territorial de la Provincia o, en su defecto, dentro de las Normas subsidiarias y complementarias de planeamiento de ámbito provincial hoy vigentes.

Si Valencia lograse tener valorado el medio físico de toda la Provincia, con las restricciones de uso en cada área, y, por otro lado, realizado un amplio Catálogo de Espacios Naturales Protegibles de diferente categoría como medida cautelar, se habría dado un paso realmente importantísimo para la protección de la Naturaleza de nuestra Provincia. Así se pondría límite al caos hoy existente, que de continuar unos años tendrá como consecuencia la pérdida de nuestras riquezas naturales y el mal empleo del suelo valenciano, que está a merced de especuladores de todo tipo que, en vez de pensar en el bien social que ello representa, sólo ven la manera de enriquecerse a costa de empeorar día a día la calidad de la vida de los valencianos

Basta ya de desecar o urbanizar nuestras zonas húmedas litorales de $\tan$ alto valor, de construir en las cumbres de nuestros más queridos y tradicionales montes, de asentar industrias y urbanizaciones en las más feraces tierras agrícolas, o bien en zonas naturales de gran interés ecológico. Tampoco deben darse tales usos en espacios donde la industria pueda contaminar acuíferos superficiales o subterráneos, o bien envenenar la atmósfera por la poca capacidad dispersante de la misma en un área determinada; todo lo cual se agrava aún más por ser insuficientes, como tantas veces sucede, los sistemas de depuración empleados.

A los ciudadanos, y no sólo a la Administración, cabe el denunciar todos estos atentados contra el medio natural, así como las infracciones urbanísticas que con gran frecuencia se realizan y que, como hechos consumados, van destruyendo implacablemente la Naturaleza. Ha llegado el momento de hablar menos, de actuar más y de decir de una vez para siempre ¡BASTA! 